\title{
VALOR, NORMA Y ACCIÓN EN MIS ESCRITOS FILOSÓFICOS* \\ Con un epílogo cartesiano
}

Georg Henrik von Wright

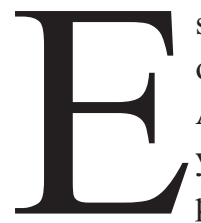

stoy profundamente agradecido a la dirección de ZIF por organizar un Autorenkoloquium en mi honor en Bielefeld en Abril de 1996. El tema del coloquio fue Acciones, Normas, y Valores. Nueve trabajos - tres por cada uno de los tópicos principales - fueron leídos y discutidos en el encuentro. Respondí a todos ellos. Luego, no menos de 30 ensayos fueron enviados para la publicación de las actas del encuentro.

En lugar de comentar cada una de esas contribuciones, he decidido escribir un ensayo sobre el lugar y el papel que han tenido en mi pensamiento los tres tópicos del coloquio. El ensayo es "autobiográfico" en el sentido que sigue el desarrollo de mis pensamientos en orden cronológico. Por ello he invertido el orden de los temas: Valor, Norma y Acción. A efectos de lograr que el articulo sea lo más autosuficiente posible, también he resumido mis puntos de vista principales y mis argumentos. Los resúmenes son, con frecuencia, enormes simplificaciones y pueden impresionar a un lector que no esté familiarizado con las fuentes como poco críticos o incluso dogmáticos.

Al seguir una perspectiva cronológica fue natural desviarme algunas veces de la división tripartita "valor-norma-acción" y hacer una breve excursión en algo que ha sido un aspecto colateral de mi labor en algunos de los otros tres tópicos. Muchos de estos resultados colaterales han surgido de la teoría de la acción. Desde mediados de los ochenta ellos se han desviado progresivamente hacia la filosofía de la mente y la metafísica de la división

* Traducción de Laura Manrique y Pablo E. Navarro. Titulo original: "Value, Norm, and Action in My Philosophical Writings. With a Cartesian Epilogue" en Meggle, G. Et al. (eds), Actions, Norms, Values. Discussions with Georg Henrik von Wright (Berlín/New York: Walter de Gruyter, 1999). En este trabajo, von Wright se refiere en más de una ocasión a diferentes exposiciones y contribuciones al coloquio celebrado en Bielefeld en Abril de 1996. Estos trabajos han sido publicados en el volumen indicado arriba. 
Cartesiana entre materia y mente. De este modo, ellos señalan un regreso a los problemas de la filosofía que fueron los que primero me atrajeron en mi temprana adolescencia. Algunas de las contribuciones en el libro siguen mis pensamientos en esa dirección. Por ello pensé que era apropiado concluir mis comentarios con un breve "epílogo cartesiano".

Agradezco especialmente a los coordinadores de las tres sesiones del coloquio, los profesores Ernesto Garzón Valdés, Werner Krawietz, y Georg Meggle. La eficiencia y la paciencia sin límites del Profesor Meggle, tanto en la planificación del coloquio como así también en la edición de sus actas, es un gesto de amistad por el que siempre estaré agradecido.

Dedico estas inspiraciones autobiográficas a los autores del volumen como una muestra de gratitud por el interés que ellos han manifestado por mis esfuerzos filosóficos.

1. En tanto que disciplina académica, la filosofía en Finlandia ha estado tradicionalmente dividida en dos ramas independientes - filosofía teórica y práctica. En la primera se ubicaban la lógica, la epistemología y la metafísica; en la segunda, la ética y la filosofía política y social. En mis tiempos de estudiante, la filosofía teórica también incluía a la psicología.

Elegí estudiar filosofía teórica, y mi principal motivación fue, hasta donde puedo recordar, que había oído del Profesor Eino Kaila, quien estaba casado con una prima de mi madre. Como consecuencia de mi elección nunca estudié filosofía jurídica, moral o social. Tampoco filosofía de la historia o religión. Esto es ligeramente irónico, considerando que una parte principal de mi trabajo en filosofía, y ciertamente la parte que es mejor conocida, ha sido en temas de dimensiones "prácticas" - y por ello caen en una disciplina en la que nunca logré una formación profesional en absoluto.

2. La influencia de Edward Westermarck era aún fuerte en el ambiente intelectual en el que crecí. Él había sido Profesor de Filosofía Práctica en Helsinki, a comienzo del siglo, y fue luego Profesor de Filosofía en la Abo Academy y de sociología en la Universidad de Londres. Él es uno de los más grandes académicos que mi país ha producido y su influencia sobre opiniones seculares en cuestiones de moral y religión ha sido profunda. Por tanto, fue mas o menos una cuestión obvia que en mis primeras, y no profesionales, reflexiones sobre cuestiones relativas al valor, implícitamente abrazase el subjetivismo y el relativismo ético en el que Westermarck había sido un campeón.

En algún sentido - tal vez no fácil de hacer explícito - he permanecido fiel a esos primeros puntos de vista. Aquello que llamaría "genuinas" valoraciones expresan la aprobación o desaprobación de un objeto por parte 
de un sujeto. Como tales, las valoraciones no son verdaderas ni falsas. La valoración de un objeto por un sujeto puede diferir de las valoraciones que otro sujeto hace del mismo objeto. Pero el enunciado "Yo apruebo esto", a diferencia de la "misma" aprobación, es verdadero o falso. En este sentido, los enunciados evaluativos tienen una ambigüedad sistemática similar a los enunciados normativos (deónticos). (Cf. Más abajo, II, 3)

Westermarck habría dicho que las valoraciones expresan sentimientos de aprobación o desaprobación - y alguien podría desear decir que ellos son actitudes expresivas de aprobación o desaprobación. Pero la expansión de la formula más breve antes indicada me parece inútil o controvertible.

3. Westermarck también introdujo la pregunta acerca de cómo distinguir la aprobación o desaprobación moral de otras clases de valoraciones. Hay que reconocer a Westermarck el merito de ver un problema aquí. Otros partidarios del "emotivismo" han tendido a ignorarlo. Por ejemplo, Stevenson en su libro, que fue influyente en su momento, Ética y Lenguaje.

La respuesta de Westermarck ${ }^{1}$ a su pregunta fue que la aprobación moral es imparcial (desinteresada) y, en un cierto sentido, universalizable. Estas ideas tienen reminiscencias de la ética de Kant y también de la Regla de Oro de la moralidad cristiana - dos autoridades en cuestiones morales que, por otra parte, Westermarck había censurado duramente.

Los dos rasgos mencionados no entran en conflicto con la subjetividad y el relativismo de las valoraciones morales. Pero ellos introducen una pregunta que Westermarck nunca consideró: ¿Qué naturaleza lógica tiene la afirmación de acuerdo con la cual de la imparcialidad y la universalizabilidad surge aprobación o desaprobación moral? ¿Se trata de un enunciado descriptivo acerca del uso del lenguaje moral? En este caso, difícilmente podría ser adecuado para todas las sociedades a lo largo de su historia. ¿O es un enunciado evaluativo o normativo, que nos urge a considerar a la moral en una cierta luz? En este caso, la caracterización de Westermarck es una contribución a lo que se llama ética normativa. Ciertamente no intentaba ser eso. Pero pienso que así debemos entenderlo.

La primera contribución que hice a la teoría ética fue un artículo del principio de 1950 "Om moraliska föreställningars sanning” (Acerca de la verdad de las ideas morales) $)^{2}$. El titulo era una alusión consciente a

${ }^{1}$ Westermarck (1924). Las emociones morales, decía Westermarck, “ no son influenciadas por la relación particular que tienen con aquellos que se encuentran inmediatamente afectados por los actos en cuestión y por aquellos que ejecutan esos actos" Y el sujeto que enuncia un juicio moral "siente que éste sería compartido por otras personas si ellos conociesen el acto y todas las circunstancias involucradas tan bien como él mismo"

${ }^{2}$ Von Wright (1954a), pp. 48-74 
una famosa conferencia de Axel Hägerström, la "contrapartida" sueca del finlandés Westermarck. El articulo contiene no sólo lo que aun me parece una interpretación moderadamente buena del núcleo de las dos "grandes" filosofías morales, sino también un intento de "rescatar" al subjetivismo valorativo de Westermarck y al nihilismo valorativo de Hägerström de los pantanos de la anarquía moral y el egoísmo subjetivista. También hice uso de ideas que aprendí de Wittgenstein. Argumenté que muchos, tal vez la mayoría, de enunciados en la esfera moral subsumen casos individuales bajo estándares (criterios) aceptados de bondad o maldad y por eso son, en un sentido relativo, verdaderos o falsos. Sin embargo, los estándares mismos no pueden ser evaluados desde el punto de vista de la verdad.

4. Pasaron otros diez años antes de que presentase al público un aporte mayor a la filosofía del valor. Éste fue mi libro The Varieties of Goodness, basado en las Conferencias Gifford que había dado en St. Andrews ${ }^{3}$. Fue publicado en 1963. Inicialmente, deseaba llamarlo "Prolegómenos a la Ética" pero, como es bien conocido, este titulo ya había sido usado (Thomas H. Green: Prolegomena to Ethics, publicado de manera póstuma por A. C. Bradley).

Una idea en Varieties, que aun me atrae de manera intensa, es que las nociones centrales para la vida moral de un individuo, tales como bueno, o malo, virtud y vicio, justicia e injusticia, son conceptos en busca de un significado. Aunque son familiares en nuestro uso cotidiano, ellas son al mismo tiempo oscuras y vacilantes. Puede existir un amplio consenso acerca de cómo usarlas - pero hay también bastante desacuerdo y controversia acerca de sus aplicaciones a casos individuales. Así, en las "intelectualizadas" y tardías etapas en la historia de una civilización deviene tarea del filósofo el reflexionar sobre el significado de las ideas morales. La tarea no es descriptiva. Pero tampoco es una cuestión de mera estipulación. La actividad del filósofo de imponer un significado tiene una finalidad práctica. Pretende ser una guía acerca de cómo juzgar y evaluar la conducta de los individuos (incluyendo la conducta del propio filósofo). Así es como entiendo a la ética normativa. "Normativo" aquí no significa "legislación". La ética no puede imponer obligaciones o prohibiciones. Pero puede proponer estándares de evaluación de acuerdo con los que las obligaciones y las prohibiciones pueden ser impuestas (o removidas) por una autoridad moral o jurídica esclarecida.

En Varieties hice una contribución modesta para satisfacer mi propio anhelo por el "significado de la moral". Puede decirse que iba en la misma

\footnotetext{
${ }^{3}$ Von Wright (1963a)
} 
línea de las de Westermarck en que trataba de combinar elementos Kantianos con la idea Cristiana del cuidado por el bien de nuestro prójimo.

Consideraba al Principio de Justicia como el centro de la moral. El deber moral es actuar conforme con ese principio por lo que he llamado un motivo moral, viz el deseo de conseguir para todos un bien que una acción similar de nuestros prójimos nos produciría a nosotros. La voluntad moral está más allá del egoísmo y el altruismo - una voluntad desinteresada e imparcial de justicia. Trata a nuestro prójimo como si su bienestar fuese nuestro y nuestro bienestar fuese suyo. Tener esta actitud es amar al prójimo como a uno mismo. Ésta continúa siendo, en pocas palabras, mi posición.

5. Después de Varieties intenté en algunas ocasiones conectar la idea de moralidad con la de racionalidad, o la idea de una vida de acuerdo a la razón.

Un primer intento en esta dirección fue un articulo, Rationality: Means and Ends, de $1986^{4}$. En este articulo examiné las dos formas de racionalidad que Max Weber distinguió con los nombres Zweckrationalität y Wertrationalität. Aristóteles había dicho que sólo los medios pueden ser objetos de deliberación, y que no se puede deliberar acerca de los fines ${ }^{5}$. Hice un esfuerzo para mostrar en qué sentido es posible una deliberación acerca de fines, y lo hice relacionando los problemas con otra idea de Aristóteles, la idea de prudencia (phronesis) o "sabiduría práctica". No estaba de acuerdo con la definición de prudencia de Sir David Ross ${ }^{6}$ como "el conocimiento de cómo asegurar los fines de la vida humana". Esto no es lo que significaba para Aristóteles cuando la caracterizaba como "un verdadero y razonado estado de capacidad para actuar con consideración a las cosas que son buenas o malas para los hombres"?.

En un trabajo inédito reciente traté de conectar la idea de prudencia con la de identidad personal. La identidad de una persona consiste en su conocimiento de quién es, a qué lugar pertenece, qué puede hacer y qué es lo que está más allá de su capacidad, qué tiene derecho a demandar y exigir de otros, y los otros a él. La identidad de una persona o su autoconocimiento, sostenía, es incompleto, deficiente, a menos que él advierta la simetría de la relación entre los seres humanos con respecto a cómo sus acciones afectan mutuamente sus bienes (bienestar). Enclavada en la posesión de autoconocimiento está una actitud moral hacia nuestro prójimo.

\footnotetext{
${ }^{4}$ Von Wright (1986), pp. 57-71

${ }^{5}$ Aristóteles, Ética a Nicómaco, III, iii, 11

${ }^{6}$ La Ética a Nicómaco de Aristóteles, traducción e introducción por Sir David Ross. The World"s Classics, Oxford University Press, 1953, p. 142

${ }^{7}$ Ética a Nicómaco, VI, v, 4.
} 
Puede ser verdadero decir que mis esfuerzos por conectar, en un nivel conceptual, la moralidad con la sabiduría práctica (prudencia), la vida según la razón y el autoconocimiento (identidad) esclarecido, refleja una cierta tensión entre tendencias subjetivistas y objetivistas en mi pensamiento acerca de los valores. Es también verdad que la "atracción objetivista" se ha hecho más fuerte con los años. Pero continúo considerando a esto como completamente compatible con el subjetivismo y relativismo en ética que, en mis años de formación, aprendí de Westermarck. Es por ello que advierto con algún desánimo el regreso al objetivismo y las teorías absolutistas del valor en las últimas décadas. En algunos lugares, ellas han sido acogidas como un remedio para el caos de valores o vacío que se ha producido luego de la secularización de la sociedad y la decadencia de la moral tradicional. Pero creo que esto es un error.

6. En 1952 publiqué un articulo con el titulo On the Logic of some Axiologic and Epitermologic Concepts ${ }^{8}$. Fue mi primer intento de desarrollar una teoría lógico-formal del valor. La inspiración para escribirlo tuvo poco que ver, si es que tuvo algo, con el interés en ética o teoría general del valor. Su fuente fue más bien mi "invento", poco tiempo antes, de la lógica deóntica. Ésta fue una teoría formal del discurso normativo - y entonces buscaba una contrapartida en el discurso evaluativo. Pero el intento resultó en un fracaso. Tengo dudas acerca de si alguien, además del autor del trabajo, ha explorado alguna vez sus más bien arduos argumentos formales. Y no estoy seguro que siga suscribiendo las intuiciones lingüísticas que el artículo trata de formalizar.

En los años subsiguientes mi interés fue capturado por otra noción evaluativa, la preferencia. Preferir algo a otra cosa es valorar o colocar la primera cosa en un lugar superior a la segunda. La relación de preferencia es irreflexiva y asimétrica. ¿Es también transitiva?, y ¿es conexa, i.e. es de tal manera que si una primera cosa es preferida a una segunda, entonces cualquier otra cosa es o bien preferida a la segunda o bien es preferida la primera a la tercera? Tal vez la cuestión tenga más de una respuesta. Mis pensamientos sobre estas cuestiones resultaron en The Logic of Preference ${ }^{9}$, publicado en 1963, el mismo año que The Varieties of Goodness y Norm and Action.

A diferencia de lo que ocurrió en 1952, este intento de crear una lógica formal del valor no fue un fracaso - aun a pesar de que puede decirse que cubre sólo una parte de un vasto campo. Finalmente había encontrado en la teoría del valor una contrapartida para la lógica deóntica en la teoría de

\footnotetext{
${ }^{8}$ Von Wright, (1952a), pp. 213-234

${ }^{9}$ Von Wright (1963b)
} 
las normas. También propuse un nombre "paralelo" para la nueva creación, Lógica Prohairética. A diferencia de lo que ocurrió con su contrapartida en la teoría de las normas, este nombre no tuvo amplia aceptación.

A principios de los 1970, regresé una vez más al tópico de la preferencia y escribí un artículo The Logic of Preference Reconsidered ${ }^{10}$. Es un artículo difícil, y los argumentos están muy comprimidos y ajustados. Contiene, sin embargo, algunas nuevas ideas y una ulterior elaboración de algunas viejas ideas - y puede aun ofrecer algo valioso. Una de sus principales ideas consiste en distinguir dos nociones de indiferencia entre alternativas: una que significaría simplemente "inexistencia de preferencia por una u otra alternativa" y la otra, más fuerte, que significaría "de rango equivalente" o "de igual valor".

Antes de abandonar este tópico, debo mencionar que mis ideas acerca de una lógica de la relación de preferencia habían sido anticipadas por Sören Halldén en su libro, The Logic of "Better", publicado en $1957^{11}$. Conocía de su existencia pero no estaba familiarizado con su contenido cuando, apresuradamente, escribí The Logic of Preference. No se me había ocurrido que "mejor" es (casi) otra palabra para "preferido". Este es un ejemplo, entre otros, de mi fracaso en advertir y reconocer debidamente los trabajos relacionados de mis contemporáneos. Pronto tendré más que decir acerca de esto. (Abajo, II, 2)

Los pensamientos de Halldén cayeron en suelo fértil. Su discípulo Bengt Hansson y, siguiendo a él, Peter Gärdenfords hicieron importantes contribuciones a un ulterior desarrollo de una teoría formal de la preferencia y en no menor medida al debate generado por el denominado Teorema de la Imposibilidad de Arrow.

\section{II}

1. Mi interés en las normas tiene un origen muy diferente a mi interés en las cuestiones del valor. Este último puede decirse que ha sido una parte y parcela de mi atracción inicial hacía la filosofía. En mis días de pre-estudiante no sólo estaba imbuido con el espíritu del relativismo y el subjetivismo ético de Westermarck, sino también había sido un lector entusiasta de Schopenhauer, Nietzsche y Guyau. Sin embargo, la maduración de ese rasgo de mi carácter filosófico llevó un largo tiempo (Arriba, I, 1).

Mi ocupación profesional en lógica me llevó directamente a las normas. Una vez que concluí mi trabajo doctoral sobre la inducción y la probabilidad, comencé a interesarme en la Entscheidungsproblem del cálculo de pre-

\footnotetext{
${ }^{10}$ Von Wright (1972), pp. 140-169

${ }^{11}$ Halldén (1957)
} 
dicados. Deseaba mostrar que la idea de Wittgenstein, expresada en el Tractatus, de la verdad lógica como una tautología podía extenderse más allá de la lógica proposicional. Esto exigía una nueva teoría de los cuantificadores. Y un día se me ocurrió, "prácticamente desde la nada", que las palabras modales "posible", "imposible" "necesario" están mutuamente relacionadas del mismo modo en que lo están los cuantificadores "alguno", "no" y "todos". No conocía nada de lógica modal entonces - pero me puse valientemente a la tarea de construir una. Era consciente del uso por Lukasiewicz de las ideas modales para su lógica polivalente, y pronto descubrí al moderno líder de la disciplina, C.I. Lewis. Pero seguí ignorando lo que Carnap y Ruth Barcan habían estado haciendo justo unos pocos años antes.

Fue en el transcurso de este trabajo que - en una conversación con amigos acerca de la ética - fui impactado por la observación de que las ideas normativas de permitido, prohibido y obligatorio parecían obedecer a los mismos patrones de interdefinibilidad que las modalidades básicas y los cuantificadores. Rápidamente escribí un articulo acerca de esta observación y lo envié a Mind, donde apareció al poco tiempo. Poco después apareció en Alemania Untersuchungen ubre den Modalkalkül de Oskar Becker ${ }^{12}$, y "Theorie des propositions normatives" de Jerzy Kalinoswski apareció en Polonia $^{13}$. Estas tres publicaciones independientes marcan el nacimiento de los estudios ahora comúnmente conocidos como Lógica Deóntica.

Tiempo después critiqué la analogía entre las ideas modales y deónticas. $\mathrm{Su}$ dificultad es la presunta identidad entre "no permitido" y "prohibido", y la consiguiente interdefinibilidad entre los operadores deónticos $\mathrm{O}$ y $\mathrm{P}$. Pienso que la analogía es insostenible y que las identidades que fueron extraídas de allí dependen de una confusión entre normas genuinas y las proposiciones normativas o enunciados acerca de la existencia o inexistencia de normas.

Desde Norm and Action (1963) ${ }^{14}$ había tratado a las obligaciones y las permisiones, $\mathrm{O}$ y $\mathrm{P}$, como dos categorías normativas básicas, siendo la prohibición definida como la obligación de omitir. Pero pienso que es correcto decir que una mayoría de lógicos deónticos continúan apegados a la tesis de la interdefinibilidad. Ésta no es la única posición en lógica deóntica en la que con el correr del tiempo he venido a integrar la minoría - en algunos casos tal vez una minoría de una única persona.

¡Es, en verdad, un pensamiento irónico que la idea de la cual la lógica deóntica surgió fue de hecho un error!

\footnotetext{
${ }^{12}$ Becker (1952)

${ }^{13}$ Kalinowski (1953), pp. 17-53

${ }^{14}$ Von Wright (1963c)
} 
2. Mucho de mi trabajo en lógica deóntica lo hice ignorando a mis predecesores y contemporáneos. Cuando me embarqué en lógica modal pensaba que estaba entrando a un terreno más o menos virgen. Esto era verdad, en el mejor de los casos para la lógica deóntica. Pero incluso ella tiene unos ancestros, que investigaciones posteriores han traído a la luz.

Una ignorancia similar ha sido característica de mis ocupaciones con el Entscheidungsproblem. Cuando en 1948 presenté una solución al problema de los predicados monádicos ${ }^{15}$, no sabía que, unos pocos años antes, Quine había publicado una solución sustancialmente similar ${ }^{16}$. (Presumiblemente ambos ignorábamos el hecho de que décadas antes Behman había resuelto nuestro problema). Y cuando más tarde resolví el problema de lo que he llamado “doble cuantificación”, el comentarista en Journal of Symbolic Logic (Ackerman) señaló que mi solución podía ser obtenida de los anteriores resultados de Gödel, Kalmár y Schütte ${ }^{17}$.

¿Qué se debe pensar acerca de tal ignorancia? Cuándo se entra a un nuevo campo de investigación y uno piensa que tiene una solución "original” al problema en ese terreno, ¿no sería correcto que el primer paso sea familiarizarse con la literatura y averiguar si el problema acaso ya ha sido resuelto? Si hubiese seguido esta receta, hubiese evitado muchos aliados ciegos y, posiblemente, algunos errores. Pero también me ha parecido que una nueva solución a un problema ya resuelto puede ser valiosa tanto porque puede haberse obtenido a partir de un nuevo enfoque como porque puede dirigir futuras investigaciones hacia una nueva dirección. También es una verdad acerca de mi carácter que cuando me surgen nuevas ideas, el trabajar en ellas absorbe por un tiempo todas mis energías y cierra mi mente a las influencias externas. (Simplemente las dejo afuera). Y cuando termino con un problema, a menudo pierdo interés en él y, por consiguiente, no me siento ansioso para excavar en su prehistoria.

Sin embargo, no siempre ocurre así. Algunas veces mi curiosidad es provocada. ¿Qué es lo que otros tenían para decir acerca del mismo tópico? Entonces, a menudo he hecho una lectura comprensiva y ello puede haber resultado en añadir comentarios, asintiendo o discrepando, a un manuscrito que todavía se encontraba en status nascendi. Los comentaristas han dicho algunas veces que he sido "influenciado" por alguno de los autores a los que me refiero. Ello no ha ocurrido normalmente, y cuando así sucedió, lo he dicho explícitamente. He reconocido la influencia de Elizabeth Anscombe y Charles Taylor en mis ideas en Explanation and Understanding. Por otra

\footnotetext{
${ }^{15}$ Von Wright (1947)

${ }^{16}$ Quine (1945)

${ }^{17}$ Journal of Symbolic Logic 17 (1952), pp. 201 y ss.
} 
parte, difícilmente pueda encontrarse alguna influencia de mis lecturas en la tradición alemana al escribir ese libro. Sólo después descubrí que $E \& U$ podía ser visto como un intento de "colmar la laguna" - que era muy extensa al momento de publicarse el libro - entre una tradición analítica AngloAmericana y una tradición hermenéutica Europea-Continental.

3. El filósofo sueco Ingemar Hedenius fue, si no el primero en advertir, al menos el primero en explotar críticamente la distinción entre normas $\mathrm{y}$ enunciados que dicen que tales y tales normas existen ${ }^{18}$. Puede decirse que la distinción está oculta por nuestro lenguaje ordinario. Un enunciado deóntico, por ejemplo "No puedes estacionar tu automóvil aquí,, puede ser usada para prohibir $o$ para informarle a alguien de las regulaciones que existen relativas al estacionamiento. Si el enunciado es usado de la primera manera, es usado (interpretado, comprendido) prescriptivamente, si se usa de la segunda manera, es usado descriptivamente.

Una opinión tradicional es que las normas no tienen valores de verdad. Esta opinión me parece indisputable, si se comprende de manera tal que significa que los enunciados deónticos, interpretados prescriptivamente, no expresan proposiciones verdaderas o falsas.

Los que atribuyen valores de verdad a las normas, como por ejemplo algunas doctrinas de "derecho natural", me parecen culpables de una confusión entre normas y proposiciones normativas. La plausibilidad de estos puntos de vista depende de los criterios que utilizan para la existencia de normas.

Si las normas no tienen valores de verdad, ¿cómo puede haber relaciones lógicas tales como la contradicción y la implicación (consecuencia)?¿Es posible una lógica deóntica?

Por una parte, asumí, ingenuamente, que el mero hecho de que uno pueda construir un calculo formal con reglas de inferencia y axiomas intuitivamente plausibles y adecuados era suficiente para mostrar que la respuesta a la pregunta era afirmativa. O aun para mostrar - como dije en el Prefacio a mi libro Logical Studies (1957) - que la lógica se extendía más allá de la verdad $^{19}$. Y esto me pareció un logro de considerable interés filosófico.

Pero mi juicio fue ciertamente demasiado apresurado.

Por otra parte, una estructura sintáctica de enunciados deónticos y sus compuestos moleculares, tales como mi sistema de 1951, podría, de acuerdo a una interpretación descriptiva de los enunciados, ser una lógica de

\footnotetext{
${ }^{18}$ Hedenius (1941)

${ }^{19}$ Von Wright (1957), p. vii
} 
proposiciones normativas. ¿Era esto a lo que equivalía realmente la lógica deóntica?

En Norm and Action (1963) me sentía cercano a esta posición. Pero también me sentía confundido.

4. En el sistema de 1951 se puede probar la formula $\mathrm{Pp} \vee \mathrm{O} \neg \mathrm{p}$. Dice que cualquier estado de cosas tiene un status deóntico, permitido o prohibido. Un orden normativo o sistema en el que es eso verdad es completo o "sin lagunas". También se pueden probar las fórmulas $\neg(\mathrm{Op} \wedge \mathrm{O} \neg \mathrm{p}) \mathrm{y}$ $\neg(\mathrm{Op} \wedge \mathrm{P} \neg \mathrm{p})$. La primera dice que ningún estado de cosas es tal que él y su contradictorio son obligatorios; la segunda dice que no puede ser el caso que lo contradictorio de un estado de cosas obligatorio esté permitido. Un orden normativo en el que esto es verdadero es consistente o libre de contradicciones.

Interpretado como una lógica de proposiciones normativas, el sistema de 1951 sería válido sólo para aquellos sistemas normativos que posean las "propiedades perfectas" de no tener lagunas ni contradicciones. Pero parece un hecho notorio que, por ejemplo, muchos órdenes jurídicos contienen tanto lagunas como normas mutuamente contradictorias.

Kelsen pensaba que los órdenes jurídicos "por una necesidad de la lógica de las normas" son completos y consistentes. Por consiguiente, él fue, por un breve tiempo, un entusiasta de la lógica deóntica, dado que le parecía probar dos de las ideas meta-jurídicas características de su pensamiento ${ }^{20}$. Pero estaba equivocado. La "moraleja lógica" que se sigue de la cuestión de facto de la existencia de lagunas y contradicciones en algunos órdenes normativos es que el sistema de 1951 no puede ser la única lógica correcta de las proposiciones normativas. Y, en vista del hecho de que las normas mismas no son verdaderas ni falsas, era al menos dudoso que este sistema pudiese pretender válidamente ser siquiera una lógica de normas.

5. Los primeros en ver claramente la necesidad de distinguir entre una lógica de proposiciones normativas y una lógica de normas (una lógica deóntica "real") fueron, pienso, Carlos Alchourrón y Eugenio Bulygin, coautores de su clásico Normative Systems ${ }^{21}$. Unos años antes de la aparición del libro, Alchourrón hizo su primer intento de construir una lógica de proposiciones normativas - que él llamaba "Lógica Normativa" - para distinguirla de una lógica de normas ${ }^{22}$. Es un gran merito de los dos autores

\footnotetext{
${ }^{20}$ Acerca de la posición de Kelsen y mi encuentro con Kelsen en 1952, véase mi articulo "Is and Ought". Von Wright (1985 a), pp. 263-281.

${ }^{21}$ Alchourrón/Bulygin (1971)

${ }^{22}$ Alchourrón (1969)
} 
haber comprendido esta doble tarea de un estudio lógico de las normas. Me ha llevado casi treinta años ver su entera significación. Y todavía no ha sido apreciada por todos los lógicos y filósofos.

6. Luego de Norm and Action continuó atormentándome la pregunta “Es posible una lógica de normas?”. Pero ahora pienso que finalmente soy capaz de justificar una respuesta afirmativa. Trataré de resumir brevemente mi última posición ${ }^{23}$.

En la interpretación descriptiva de los enunciados deónticos, sus compuestos moleculares son enunciados que expresan proposiciones verdaderas o falsas. Los compuestos son, en otras palabras, veritativo-funcionales, y tienen significado descriptivo. Pero conforme a la interpretación descriptiva de los enunciados deónticos, los compuestos moleculares formados por ellos con la ayuda de las conectivas no expresan normas, y no tienen significado prescriptivo. Las conectivas, en otras palabras, no se aplican a las normas, i.e. a enunciados deónticos prescriptivamente interpretados (Hay aparentemente unas pocas excepciones a esto. En otro lugar las he denominado "accidentes semánticos". No necesitamos ocuparnos de ellos aquí)

Se sigue de lo que se ha dicho que no es posible construir una lógica de normas en la forma de una estructura sintáctica de variables proposicionales, conectivas y operadores deónticos. Los enunciados deónticos en esa estructura sólo pueden recibir interpretación descriptiva. Esto significa que la estructura es, en el mejor de los casos, una lógica de proposiciones normativas y no una lógica de normas.

¿No provoca esto, si se acepta el argumento, un hundimiento de toda la tarea de construir una lógica deóntica "real"? Creo que no. Hay otro enfoque del problema además del sintáctico. Lo llamaré enfoque semántico. Consiste en lo siguiente:

Diré que un conjunto de normas es consistente si y sólo si la conjunción de todas las cosas (contenidos normativos) que las normas del conjunto declaran obligatorias junto con cualquiera de las cosas que ellas permiten es un estado de cosas susceptible de ser alcanzado, i.e. es algo que puede obtenerse como un resultado o consecuencia de la acción humana. Un conjunto que no es consistente es inconsistente o auto-contradictorio.

Conforme a esta definición, para recurrir a un ejemplo muy simple, las dos normas $\mathrm{Op}$ y $\mathrm{O} \neg$ p constituyen un conjunto inconsistente. Esto es así

\footnotetext{
${ }^{23}$ El enfoque que doy aquí sigue el de mi artículo "Is There a Logic of Norms?” (1991) y su versión revisada, incluída en mi colección Normen, Werte und Handlungen (1994). Véase también "Ought to be - Ought to do" en el presente volumen [El autor se refiere aquí a "Ought to be - Ought to do" en Meggele, G et al (eds), Actions, Norms, Values. Discussions with Georg Henrik von Wright (Berlín/New York: Walter de Gruyter, 1999]
} 
porque la conjunción de sus contenidos, $\mathrm{p} \wedge \neg \mathrm{p}$ no es un estado de cosas susceptible de ser logrado.

Que $\mathrm{p} \wedge \neg \mathrm{p}$ son inconsistentes es un hecho de la lógica. Pero, ¿por qué debemos decir que $\mathrm{Op}$ y $\mathrm{O} \neg$ p son mutuamente inconsistentes? Pienso que no podemos dar una respuesta satisfactoria a menos que consideremos el propósito o racionalidad de las normas y la actividad normativa. Una autoridad normativa (un legislador) que se involucra en que sus sujetos normativos hagan p y, al mismo tiempo, $\neg$ p, estaría “pidiendo la luna”, demandando algo que no puede ser, y por ello comportándose de manera irracional. Pero no hay nada irracional en el hecho de que una autoridad procure que $\mathrm{p} \mathrm{y}$ otra autoridad que $\neg$ p. Al llamar mutuamente inconsistentes a Op y $\mathrm{O} \neg \mathrm{p}$ se presupone la identidad de la autoridad normativa y de la ocasión para la acción.

Luego introducimos la noción de norma negación. La negación de la obligación Op es una permisión $\mathrm{P} \neg \mathrm{p}$. Y la negación de una permisión $\mathrm{Pp}$ es una obligación $\mathrm{O} \neg$ p, i.e. la prohibición de p. Esto responde al entendimiento ordinario del sentido prescriptivo de las frases "no-obligatorio" y "no-permitido".

Podemos ahora definir la noción de implicación normativa. Diré que un conjunto consistente de normas implica a otra norma cuando, y sólo cuando, al añadir a ese conjunto la norma negación de esa otra norma convierte al conjunto en inconsistente.

Consideremos un ejemplo muy simple: Op implica a Pp. El conjunto formado por Op y $\mathrm{O} \neg$ p es inconsistente. De allí que la obligación (“deber") implique a la permisión (“poder”).

La lógica deóntica, tal como la veo ahora, descansa en la posibilidad de definir las nociones de consistencia normativa e implicación de manera que estén de acuerdo con nuestras intuiciones. Me llevó unos treinta años alcanzar esta posición. No es probable que cambie nuevamente, excepto tal vez en detalles. Las semillas de mi punto de vista están ya en Norm and Action. En los 1980 mis puntos de vista se radicalizaron y se acercaron más al "nihilismo" de Hägerström y al de Kelsen en sus últimos años. Como ellos llegué a sostener que, en el sentido tradicional, no se pueden obtener genuinas relaciones lógicas entre normas. Pero, a diferencia de ellos, pensaba que era posible "trascender" la lógica y aplicar las nociones de consistencia y consecuencia lógica más allá de los límites de la verdad y la falsedad, reivindicando así las intuiciones originales que están detrás de mi ensayo de 1951.

7. En mi trabajo introductorio al simposio de Bielefeld me ocupé de otros problemas centrales que me han intrigado a lo largo de los años. Ellos tienen 
que ver con el tema controvertido de las normas hipotéticas y la importante distinción entre lo que he llamado el "deber de obligación" y el "tener que de la necesidad práctica”. No repetiré aquí lo que tenía para decir acerca de estos tópicos sino que me remitiré a la versión publicada de este trabajo ${ }^{24}$.

\section{III}

1. Mi interés por la acción fue una consecuencia inmediata de la invención de la lógica deóntica.

Las "cosas" que las normas declaran obligatorias, permitidas o prohibidas son, hablando genéricamente, acciones o "estados de cosas susceptible de hacerse" (doable state of affairs), es decir estados que pueden ser realizados, producidos como resultados o consecuencias de una acción. Una acción del tipo de las de realizar (achivment type) tiene lo que llamo un aspecto "interno" y un aspecto "externo". Este último consiste en cambios (o no-cambios) en el mundo físico - en primer lugar en las reacciones que se producen en el cuerpo del agente. El primero es algo "mental", la intención o la voluntad del agente de lograr un cambio o asegurar que algo permanezca sin modificarse.

Mi preocupación por la acción se concentró, en primer lugar, en el aspecto externo, un estudio del cambio. Ello se reflejó en mi primer intento, en Norm and Action, de crear una Lógica de la Acción. Pero pronto me interesé también en el aspecto mental o "volitivo" de la acción. Esto resultó en lo que más tarde se convirtió en tema principal de mi teoría de la acción, es decir la explicación de la acción. Me ocupé de ella en Explanation and Understanding $(1971)^{26}$ y trabajos posteriores.

Sea "p" una representación del estado de que una cierta ventana está abierta. Este estado puede darse como resultado de dos diferentes acciones. Una es la acción de abrir la ventana, esto es cambiando su estado de cerrada, $\neg p$, a abierta, $p$. La otra es la acción de prevenir que la ventana se cierre, por ejemplo porque alguien más está tratando de cerrarla.

Las dos acciones pueden diferenciarse en su "status deóntico". La primera puede ser permitida y hasta obligatoria, la segunda estrictamente prohibida. Pero esta diferencia no puede ser expresada en la lógica deóntica "clásica". Estas expresiones requieren un simbolismo para el cambio. Digamos que " $\neg p T p$ " representa el pasaje de que la ventana está cerrada a que

${ }^{24}$ El trabajo fue también publicado, bajo el titulo "Ought to be-Ought to do" en Garzón Valdés et al. (1997), pp. 427-435. (Añadido de los traductores: en el texto von Wright se refiere a: "Ought to be - Ought to do" en Meggele, G et al (eds), Actions, Norms, Values. Discussions with Georg Henrik von Wright (Berlín/New York: Walter de Gruyter, 1999))

${ }^{26}$ Von Wright (1971). 
la ventana está abierta, y que " $p T p$ ” representa que la ventana permaneció abierta. Si estos dos eventos son pensados como resultado de una acción, entonces " $O(\neg p T p)$ " representa la obligación de abrir la ventana, y " $O \neg($ $p T p$ )" a la prohibición de evitar que ésta sea cerrada. (Esto es solamente un primer paso a un simbolismo completo, pero será suficiente para los presentes propósitos)

El corolario de estas observaciones fue que un desarrollo completo de la lógica de normas tiene que "apoyarse en los hombros" de una lógica de la acción, la cual a su vez tiene que construirse sobre una lógica del cambio. Pero era preciso construir esos fundamentos ausentes de la lógica deóntica. En Norm and Action me embarqué en esa tarea.

Pensé que debía comenzar desde cero. En lo que concierne a la Lógica de la Acción estaba presumiblemente en lo correcto. No conozco de ningún esfuerzo sistemático anterior en esta misma dirección. Y después de más de treinta años sigo teniendo la impresión de que el estudio lógico formal de la acción permanece relativamente poco desarrollado y es algunas veces indebidamente descuidado.

Mi trabajo en la lógica de la acción, luego de Norm and Action, intentaba construir una teoría axiomática para los cuatro tipos de acciones atómicas o elementales que tengan la naturaleza de poder ser realizadas [the nature of achievements], viz estos tipos son producir, evitar, mantener, y destruir y las correspondientes omisiones de sus realizaciones. Mi contribución más reciente es el trabajo "Action Logic as a Basis of Deontic Logic" de $1988^{27}$. También pienso que será el último.

Otros que han trabajado en este campo, en general, no han construido sobre la fundamentación "cuatripartita" de los modos elementales de acción. Un interesante enfoque de naturaleza, en algún sentido, un tanto diferente es el de la lógica dinámica de Krister Segerberg. Otro es la combinación de ideas normativas y de acciones de Nuel Belnap en su See-To-It-That. Me alegró encontrar a ambos escritores entre los contribuyentes de este volumen.

En el nivel básico de mi nueva construcción, la lógica del cambio, las cosas se entienden en algún modo diferente. Su origen es otro ejemplo de mi frecuente ignorancia de los trabajos realizados paralelamente por otros lógicos. Mi lógica del cambio terminó siendo un fragmento pequeño del trabajo en la lógica del tiempo, que había sido iniciado algunos años atrás por Arthur Prior ${ }^{28}$. Empecé con una teoría de la conjunción asimétrica "and next". Ella captura transiciones (progresos, cambios) de un estado a otro

\footnotetext{
${ }^{27}$ Von Wright (1988)

${ }^{28}$ Sobre esto y su relación con algún trabajo hecho por mi ver Prior (1987).
} 
en una medida temporal discreta. Poco después, Segerberg produjo una versión mejorada del mismo con el título "On the Logic of 'To-morrow". ${ }^{29}$ Mi siguiente objetivo fue otra conjunción asimétrica "and then". Se refiere a progresiones de una longitud no especificada en un medio temporal que no necesita ser discreto. Su axiomatización resultó ser mucho más engañosa que aquella de "and next". Me ocupé intensamente de este tema durante un trimestre en la Universidad de Pittsburgh a mediados de los sesenta. Éste fue un periodo de excepcional fertilidad en mi vida creativa. Resultó, entre otros escritos, en An Essay in Deontic Logic and the General Theory of Action (1968). ${ }^{30}$ Prior visitó Pittsburgh y dio una conferencia. Gracias a él me di cuenta de que mi trabajo con los dos asimétricos "ands" había sido mi manera solitaria, y quizás innecesaria, de abordar la lógica temporal. Pero no tomé esto como una pérdida de tiempo y energía, sino lo contrario.

Así, el estudio del cambio, que era originariamente una introducción al estudio de las normas y acciones, despertó mi interés en lo que yo llamaría la Lógica del Tiempo, antes que usar la expresión Lógica Temporal. Por algunos años continué trabajando en esta nueva dirección. Si contribuí en algo de valor original fue, quizás, el inicio de un estudio de la "división del tiempo"- el "fraccionamiento" sucesivo de un periodo de tiempo en partes más pequeñas y pequeñas en contraste con "la suma de" estos periodos en progresiones hacia el futuro y el pasado ${ }^{31}$. También me aventuré en la dimensión espacial, en algo que llame la Lógica del Espacio ${ }^{32}$. Finalmente, estas preocupaciones estimularon mi interés en varios tipos de lógica no-clásica, divergentes ${ }^{33}$. Ellas marcan, creo, el final de mi travesía en la lógica.

2. Casi de manera paralela con mis esfuerzos por basar la lógica deóntica en una lógica de la acción, desarrollé mi trabajo acerca de la inferencia práctica. La inspiración, si lo recuerdo correctamente, surgió de mi estudio de Aristóteles cuando estaba preparando The Varieties of Goodness - y un apoyo muy importante fue la obra maestra de Elizabeth Anscombe, Intention (1957).

Un argumento práctico (inferencia, silogismo) tiene dos premisas. Una es valorativa; se refiere a un fin que es codiciado (querido) por un agente. La otra es epistémica; menciona lo que el agente piensa acerca de los medios para este fin. La conclusión, finalmente, consiste en que el agente procede

\footnotetext{
${ }^{29}$ Segeberg (1967).

${ }^{30}$ Von Wright (1968).

${ }^{31}$ Von Wright (1969).

${ }^{32}$ Von Wright (1983a).

${ }^{33}$ Ver mi trabajo "Truth-Logics" (1987), pp.311-334
} 
a actuar, i.e. usa los medios en pos de alcanzar el fin. Esta es solamente una descripción inicial y superficial; hay alternativas y diferentes maneras de describir básicamente la misma estructura. Me llevó, por ejemplo, algún tiempo darme cuenta que la manera en que Anscombe entiende a Aristóteles era considerablemente distinta a la mía. ${ }^{34}$

¿Es la inferencia práctica un argumento lógicamente válido? La respuesta, por supuesto, depende de cómo se establezcan las premisas y la conclusión en términos más precisos. La cuestión clave es averiguar si hay alguna clase de conexión necesaria (implicación) entre, por un lado las finalidades del agente y los medios estimados y, por el otro lado, sus efectivos esfuerzos para lograr sus fines. En innumerables discusiones con Norman Malcolm ${ }^{35} \mathrm{y}$ Fred Stoutland, intentamos formular una versión plausible de lo que Stoutland ha llamado "el argumento de la conexión lógica". ${ }^{36}$

Un argumento práctico mira hacia el futuro en el sentido de que procede (en el pensamiento) desde el trasfondo de fines y medios $a$ la proyectada realización de la acción. Está relacionado con la predicción. Uno puede, sin embargo, invertir el orden y argumentar desde la realización de la acción hacia su trasfondo de medios y fines. Entonces, el esquema se relaciona con la explicación de la acción. Esto no significa que se pueda inferir las premisas desde la conclusión. Pero significa que uno puede referirse a las premisas como razones para la acción.

La explicación de la acción ha sido uno de los temas principales en mi pensamiento en los últimos treinta años, o más. Temo que la maduración de mis reflexiones se vio retrasada por el hecho de que durante un largo período he tendido a ver el problema a la luz prospectiva de la predicción de la acción antes que en la retrospectiva de la explicación de la acción.

3. ¿Son las razones para una acción causas de la acción? La pregunta no es acerca del uso lingüístico. Las razones para la acción son usualmente (también) llamadas “causas". Esto es correcto. Lo que me provocaba ansiedad al responder negativamente la pregunta de más arriba, era enfatizar una diferencia conceptual entre la explicación de la acción intencional en términos de razones (motivos) y la explicación de los eventos naturales en términos de causa y efecto. Por tanto, he distinguido entre explicación racional de la acción y la explicación causal de los eventos naturales.

El esquema general de la explicación causal es el nomológico-deductivo o el esquema de Leyes de Cobertura. Este subsume un par de eventos indi-

\footnotetext{
${ }^{34}$ Ver Anscombe (1989), y mi respuesta a Anscombe.

${ }^{35}$ Ver Malcolm (1989) y mi replica a Malcolm.

${ }^{36}$ Ver Stoutland (1970).
} 
viduales bajo una regla o ley, correlacionando tipos de eventos con ejemplos de sus instanciaciones. El esquema para una explicación racional es, podría decirse, una inferencia práctica invertida. Entiende a una acción individual de acuerdo al trasfondo de sus razones, i.e., los fines deseados por el agente y los medios que se tienen en cuenta para lograrlos.

En Explanation and Understanding, y en trabajos posteriores, defendí la autonomía conceptual de la explicación racional de las acciones respecto a las explicaciones causales de los eventos. Por algún tiempo argumenté que el esquema de la inferencia práctica es para las ciencias humanas lo que el esquema de las Leyes de Cobertura es para las ciencias naturales. ${ }^{37}$ Esto era una exageración - aunque creo que contiene un ápice de verdad. Mi posición era una defensa de la autonomía de las ciencias humanas (Humanidades or Gesterwissenschaften) en relación con las ciencias naturales ( $\mathrm{Na}$ turwissenschaften). Está por lo tanto en oposición a la idea de la "unidad de la ciencia" de positivistas lógicos y empiristas. Mi visión "dualista" puede ser relacionada con la distinción Erklären-Verstehen y el debate familiar en la filosofía alemana de fin de siglo, revivido después en la filosofía "hermenéutica" contemporánea. (Ver arriba II, 2)

La distinción entre explicación y comprensión, sin embargo, está lejos de ser siempre clara y algunas veces provoca confusión. Por ello, en años recientes me he concentrado en otra dicotomía que parece calar más profundamente. Es la división entre conocer la verdad y entender el sentido o significado. El conocimiento y la comprensión, diría, son las dos categorías o dimensiones básicas de la vida cognitiva del hombre - y va en detrimento de nuestra cultura intelectual haber enfatizado en el conocimiento a expensas de la comprensión.

Fue en la "Münsteraner Disputationen" con Georg Meggle en los finales de 1980 que se produjo un cambio decisivo en mi pensamiento desde la temática de la explicación-comprensión hacia la relación entre conocimiento y comprensión. Traté de defender en nuestras charlas - en contra de Meggleel punto de vista de que la comprensión no es una forma de conocimiento (Wissen) - aunque presupone conocimiento (por ejemplo, de lo que contará como una explicación inteligible de una acción en términos de sus razones). La comprensión, por decirlo así, "produce su propia verdad" en el acto concreto de conectar la comprensión mental con su objeto. Espero que todavía sea capaz de expresar mis intuiciones acerca de esta relación de una manera más clara y convincente ${ }^{38}$.

\footnotetext{
${ }^{37}$ Von Wright (1971), p.27

${ }^{38}$ Von Wright/ Meggle (1989), reimpreso en von Wright (1994).
} 
4. En Explanation and Understanding también defendí lo que algunas veces se llama una visión intervencionista o basada en la acción de las conexiones causales o nómicas en la naturaleza. La pregunta era: ¿se puede distinguir entre secuencias meramente regulares o concomitancia entre eventos - la visión Humeana de la causalidad- $y$ algún lazo más fuerte entre ellos que merezcan quizás ser llamados necesidad natural? En $E \& U$ argumenté a favor de esta distinción. En una forma muy simplificada, mi argumento puede ser presentado como sigue:

Es característico de una conexión nómica entre dos eventos, E1 y E2, que ésta apoye enunciados contrafácticos del siguiente tipo: Si E1 hubiese ocurrido en una ocasión, cuando de hecho no ocurrió, entonces E2 también hubiese ocurrido. Pero ¿cómo puede hacerse para que esto sea plausible? Mi respuesta fue: produciendo E1, en una situación en la que estamos razonablemente seguros de que ni éste ni E2 ocurrirán "por ellos mismos" o por alguna causa ya conocida, y luego advirtiendo que E2 también aparece. Esto presupone que E1 es un estado de cosas susceptible de ser producido y que dominamos la técnica de producirlo. La presuposición es normalmente satisfecha en el importante tipo de acción humana que llamamos experimento (científico).

Mi teoría puede, por tanto, también llamarse una teoría experimentalista de la causalidad. Puede ser fácilmente malinterpretada. Convierte a la causalidad en conceptualmente dependiente de la acción, pero esto no significa que cada vez que algo causa otra cosa la causa sea producida por algún agente. (Esto sólo ocurre esporádicamente)

Una objeción ha sido que la teoría se sostiene, a lo sumo, para fenómenos terrenales, accesibles a la manipulación, pero no puede reclamar validez para el universo como un todo. Se pueden responder dos cosas. Primero, que las conexiones legaliformes en la dimensión cósmica - por ejemplo relativa al origen del universo - tienen un carácter especulativo, las cuales pueden tornar a la distinción entre lo accidental y lo nómico de difícil o imposible aplicación. Y segundo, muchas leyes, que no vacilamos en sostener como verdaderas para fenómenos que se encuentran mas allá del alcance de la acción - por ejemplo en geología o en teorías de la evolución -, son en última instancia extrapolaciones de descubrimientos bajo condiciones en que la manipulación experimental era posible.

Una teoría de la necesidad natural similar a mi visión basada en la acción no es desconocida por la literatura. Es una reminiscencia del punto de vista sostenido por Collingwood y, en tiempos mas recientes, por Gasking. ${ }^{39}$ Fue gratificante para mí advertir que algunos de los participantes en este volu-

\footnotetext{
${ }^{39}$ Gasking (1954).
} 
men, Egidi, von Kutschera y Schneider, parecen simpatizar con ella - al menos "moderadamente".

5. De todos los libros que he escrito, Explanation and Understanding es quizás el más desafiante o incluso problemático. Para mí esto representó una urgencia en retomar sus temas principales - acción y causalidad - para tratar de establecer mis puntos de vista más claramente, y para modificarlos y desarrollarlos con mayor profundidad. Este esfuerzo resultó en dos nuevos libros. Uno llamado Causality and Determinism y está basado en las Conferencias Woodbridge que dí en Columbia en $1972^{40}$. El otro tiene el título de Freedom and Determination (1980) y continúa siendo, debo decir, la exposición más completa y mejor argumentada de mi posición en filosofía de la acción ${ }^{41}$. Representa un avance de mis anteriores puntos de vista gracias principalmente a la distinción entre los determinantes internos y externos de la acción. Toma en cuenta la dependencia del agente de las condiciones, instituciones, y prácticas en la sociedad de la que es miembro. De este modo el problema de la libertad humana se transforma y se convierte en un problema central de la filosofía social. Me arrepiento de no haber seguido esta línea más allá. Me hubiese llevado al vecindario de la tradición Marxista- Hegeliana y la "teoría critica" de la Escuela de Frankfurt y de Habermas.

Cuando retorné al problema de la libertad en mis Conferencias Tanner en $1984^{42}$ mis pensamientos habían ya empezado a moverse en un campo distinto de investigación, diferente al que cubre la rubrica "Acción, Norma y Valores". Pero dado que algunas contribuciones muy interesantes - de Gjelsvik, Kambartel, Schneider - a las Actas de Bielefeld siguieron mis reflexiones en esta nueva dirección, debería concluir mi exposición con un simple y corto comentario sobre ésta.

\section{IV}

1. Distinguí (arriba III, 1) entre el aspecto externo e interno de una acción. El primero es un cambio (o un no-cambio) en el mundo exterior. El segundo es la base mental de este cambio en la voluntad, intenciones, motivos, y razones de un agente, cuyas "operaciones", como decimos, dan lugar al cambio. El aspecto interno es aquí, en algún sentido, la causa del aspecto externo y el externo el "producto" (output) del interno. Así: causa mental (psíquica) - efecto físico.

\footnotetext{
${ }^{40}$ Von Wright (1974).

${ }^{41}$ Von Wright (1981).

${ }^{42}$ Von Wright (1985).
} 
También se da el caso inverso. Un evento en el mundo físico, por ejemplo un sonido, afecta un órgano sensorial y "evoca" una sensación. Aquí el suceso externo es el inicio (input) y el interno el producto. Causa Física - efecto mental (psíquico).

Esta descripción de interacción entre lo psíquico y lo físico no soluciona una cantidad de graves problemas. Desde los días de Descartes éstos han sido de central preocupación para la filosofía occidental. Descartes hizo una clara distinción entre lo mental y lo físico, mente y materia. Pero también pensaba que los dos interactúan causalmente. ¿Es realmente así? Y si lo es, ¿Cómo deberíamos entenderlo? No es una exageración decir que las posiciones metafísicas más importantes defendidas por los filósofos desde Descartes han sido intentos de responder a las anteriores preguntas. En este sentido uno puede decir que Descartes ha eclipsado el paisaje filosófico desde sus días hasta la actualidad.

Algunos de los sucesores inmediatos de Descartes negaron la interacción entre mente y materia y pensaban que su "conexión" era algo ocasionado por una voluntad divina; Leibniz hablaba de una "armonía preestablecida". Otros sugerían una solución reduccionista - ya sea una reducción materialista de la mente a la materia como Hobbes, o una idealista de la materia a la mente como en el caso Berkeley. Otros, los monistas, seguían pensando a las dos sustancias como una. Así, Spinoza en el siglo XVII y los empiriocriticistas del siglo XIX. No hace mucho tiempo el dualismo interaccionista experimentó una resurrección, desarrollándose un nuevo retorno a las proximidades de Descartes. ${ }^{43}$

2. Una reflexión seria acerca de estas cuestiones debe tomar en cuenta el papel que juega el sistema nervioso (el cerebro). Nuestra descripción inicial no dice nada acerca de esto.

Las fuerzas de la voluntad (las motivaciones) detrás de una acción no dan lugar de forma inmediata a cambios de conducta (cambios corporales) en el mundo, que son los que constituyen el primer escalón de su aspecto externo. Ellas producen cambios neuronales, los cuales, a su vez, son causalmente responsables por el aspecto de comportamiento (behavioural aspect) de la acción.

De manera similar, la afectación de un órgano sensorial por un estimulo no resulta inmediatamente en una sensación, sino en un proceso neuronal que se "transforma" desde un estado central en el cerebro en una sensación. (Por ejemplo, cuando alguien se levanta de la silla como respuesta a un golpe en la puerta).

${ }^{43}$ Cf. Popper/Eccles (1977) 
La naturaleza "misteriosa" de la transición del proceso neuronal a la sensación mental es responsable, creo yo, de la tentación de decir que la sensación y la manifestación neuronal que esta allí cuando el sujeto tiene la sensación, son en algún sentido, lo mismo o idénticas. Y hay una tentación similar en identificar el trasfondo mental de la voluntad de una acción con los estados neuronales y los procesos que son causalmente responsables de la parte conductual de su aspecto externo.

Rendirse a esta tentación es asumir una teoría de la identidad acerca de la relación cuerpo - mente. Pero la identificación resulta de la confusión.

3. Un modo de clarificar estos problemas involucra establecer un mapa detallado de las interconexiones de los tres factores principales: el mental, el neuronal y el conductual. Lo que es característico de su interconexión, como yo lo veo, son tres diferentes relaciones de prioridad conceptual. Las llamaré, epistémica, causal, y semántica.

Lo mental, diré, es epistémicamente prioritario a lo neuronal. Con esto quiero decir lo siguiente: En pos de conocer la contrapartida neuronal de los estados mentales (fenómenos) debemos ser capaces de identificar esos estados - por ejemplo escuchando un sonido, sintiendo dolor, teniendo miedo, creyendo o esperando algo - usando criterios que sean independientes de su base neuronal. Porque de otro modo uno no podría localizar los eventos neuronales entre los que uno debe buscar la contrapartida o equivalencia con los eventos mentales ya conocidos. Por ejemplo, no sabríamos nada acerca del "centro del dolor" en el cerebro, a menos que hayamos experimentado con sujetos que conocen lo que es sentir dolor como consecuencia de cierto estímulo físico que les ha sido aplicado.

Más aún, hay una prioridad causal de lo neuronal en relación con lo conductual. Esto es sencillamente una cuestión de verdad científica. Las reacciones del cuerpo que llamamos comportamiento - no así, por ejemplo, los reflejos incondicionados - son causados por impulsos que se producen en el centro motor del cerebro.

Finalmente, está lo que propongo llamar prioridad semántica de la conducta en relación con la mental. Ésta consiste, hablando rudimentariamente, en esto: que las reacciones conductuales causadas por los equivalentes neuronales de los diversos estados mentales constituyen lo que significa decir que el sujeto experimenta ("sufre") esos estados. Este compromiso semántico de lo conductual con lo mental es una relación más compleja, y por esto también más controvertida, que aquella entre lo mental y lo neuronal; y lo neuronal y lo conductual.

4. ¿Interactúan, entonces, cuerpo y mente? En algún sentido, filosóficamente no comprometido, efectivamente lo hacen. Por supuesto, los 
estímulos físicos de los órganos sensoriales evocan las sensaciones, y las razones mueven a los agentes a actuar. Descartes, quien creía en la interacción cuerpo - mente, también estaba preocupado por la cuestión de si esto era compatible con el determinismo estricto en la naturaleza. Una manera de rescatar la compatibilidad es suscribir el siguiente Principio de Clausura Causal del Orden del Mundo Físico:

Siempre que pueda decirse correctamente que un evento en la naturaleza $\mathrm{E}$ causa un evento mental $\mathrm{M}$, existe otro evento físico $\mathrm{F}$ tal que $\mathrm{E}$ causa $\mathrm{F}$ y $\mathrm{M}$ coincide temporalmente con (en todo o en parte) la duración de F. Y cuando, a la inversa, puede decirse correctamente que un evento mental $\mathrm{M}$ causa $\mathrm{E}$ en el mundo físico, existe otro evento físico $\mathrm{F}$ tal que $\mathrm{F}$ causa $\mathrm{E}$ y la duración temporal de $\mathrm{M}$ es incluida en $\mathrm{F}$.

Aceptando esta idea, que me parece plausible, uno puede dejar de hablar de la interacción cuerpo - mente y mente - cuerpo para hablar de conexiones causales entre eventos físicos únicamente. Usando una terminología ahora de moda, puede decirse que la Clausura Causal del Orden del Mundo Físico significa que las relaciones causales entre fenómenos mentales y físicos son supervinientes en el tiempo a las relaciones causales cuyos elementos son sólo fenómenos físicos.

La posición sugerida deviene tan "cercana" a la tesis de identidad psíquico - física, como es, en mi opinión, "lógicamente permisible". También es una reminiscencia de versiones de la posición conocida como epifenomenalismo.

5. La distinción cuerpo - mente, como yo veo las cosas, no es una nítida división. La prioridad epistémica de lo mental con relación a lo neuronal, y de lo conductual con relación a lo mental, significan que la mente y el cuerpo están conceptualmente entrelazadas en la base de la realidad. Sostener esta intuición ha significado colocarme bajo lo que siento como una carga Cartesiana.

También tres de los mayores esfuerzos en la filosofía de este siglo, Husserl, Heidegger y Wittgenstein, pueden, en sus diferentes modos, ser vistos como esfuerzos para escapar de las sombras de Descartes. Quizás pueda permitirme decir que he intentado seguir un camino independiente en la misma dirección.

(Trad. de Laura Manrique y Pablo Navarro) 


\section{Bibliografía}

Alchourrón (1969). Carlos E. Alchourrón: Logic of Norms and Logic of Normative Propositions. Logique et Analyse 12 (1969).

Alchourrón/Bulygin (1971). Carlos E. Alchourrón and Eugenio Bulygin: Normative Systems. Wien 1971.

Anscombe (1989). Elizabeth Anscombe: Von Wright About "Practical Inference". En Schilpp/Hahn (1989).

BECKER (1952). Oskar Becker: Untersuchungen iiber den Modalkalkül. Meisenheim 1952.

Bernardo (1988). Giuliano di Bernardo (ed.): Normative Structures of the Social World. Amsterdam 1988.

Bulygin et al. (1985). Eugenio Bulygin, Jean-Louis Gardies and Ilkka Niiniluoto (eds.): Man, Law, and Modern Forms of Life. Dordrecht 1985.

Garzon Valdes et al. (1997). Ernesto Garzou Valdes, Werner Krawietz, Georg Henrik von Wright and Ruth Zirnmerling (eds.): Normative Systems in Legal and Moral Theory, Festschrift for Carlos Alchourron and Eugenio Bulygin. Berlin 1997.

GASking (1954). D. Gasking: Causation and Recipes. Mind 63 (1954).

Hallden (1957). Soren Hallden: On the Logic of "Better". Lund 1957.

HedEnIUs (1941). Ingemar Hcdenius: Om rdtt och moral. Stockholm 1941.

Kalinowski (1953). Jerzy Kalinowski: Theorie des propositions normatives. Studia Logica 1 (1953).

Malcolm (1989). Norman Malcolm: Intention and Behavior. En Schilpp/Hahn (1989).

McmurRin (1985). Sterling M. McMurrin (cd.): Tanner Lectures on Human Values, Vol. VI. Salt Lake City 1985.

Popper/Eccles (1977). Karl Raimund Popper and John C. Eccles: The Self and Its Brain. London 1977.

Prior (1967). Arthur Norman Prior: Past, Present, and Future. Oxford 1967.

Quine (1945). Willard Van Orman Quine: On the Logic of Quantification. The Journal of Symbolic Logic 10 (1945).

Schilpp/Hahn (1989). Paul Arthur Schilpp and Lewis Edwin Hahn (eds.): The Philosophy of Georg Henrik von Wright. La Salle, 111. 1989.

Segerberg (1967). Krister Segerberg: On the Logic of "To-morrow". Theoria 33 (1967).

Stoutland (1970). Frederick Stoutland: The Logical Connection Argument. American Philosophical Quarterly 7 (1970).

Westermarck (1924). Edward Westermarck: The Origin and Development of the Moral Ideas, Vol. I, Second edition. London 1924.

Von Wright (1947). Georg Henrik von Wright: On the Idea of Logical Truth I. Helsingfors: Societas Scientiarurn Fennica, Commentationes Physico-Mathematicae, Vol. IV, no. 4, 1947.

Von Wright (1952a). Georg Henrik von Wright: On the Logic of Some Axiological and Episternological Concepts. Ajatus 17 (1952). 
Von Wright (1952b). Georg Henrik von Wright: On Double Quantification. Helsingfors: Societas Scientiarurn Fennica, Commentationes Physico-Mathematicae, Vol. XVI, no. 3, 1952.

Von Wright (1954a). Georg Henrik von Wright: Om moraliska föreställningars sanning. En von Wright (1954b).

Von Wright (1954b). Georg Henrik von Wright: Vetenskapens funktion i samhallet. Copenhagen 1954.

Von Wright (1957). Georg Henrik von Wright: Logical Studies. London 1957.

Von Wright (1963a). Georg Henrik von Wright: The Varieties of Goodness.

London 1963.

Von Wright (1963b).Georg Henrik von Wright: The Logic of Preference. Edinburgh 1963.

Von Wright (1963c). Georg Henrik von Wright: Norm and Action. London 1963.

Von Wright (1968). Georg Henrik von Wright: An Essay in Deontic Logic and the General Theory of Action. Amsterdam 1968.

Von Wright (1969). Georg Henrik von Wright: Time, Change, and Contradiction. London 1969.

Von Wright (1971). Georg Henrik von Wright: Explanation and Understanding. London 1971.

Von Wright (1972). Georg Henrik von Wright: The Logic of Preference Reconsidered. Theory and Decision 3 (1972).

Von Wright (1974). Georg Henrik von Wright: Causality and Determinism. New York 1974.

Von Wright (1980). Georg Henrik von Wright: Freedom and Determination Amsterdam 1980.

Von Wright (1983a). Georg Henrik von Wright: A Modal Logic of Place. En

Von Wright (1983b).

Von Wright (1983b). Georg Henrik von Wright: Philosophical Papers, vol. II,

Philosophical Logic. Oxford 1983

Von Wright (1985a). Georg Henrik von Wright: Is and Ought. En Bulygin et al. (1985).

Von Wright (1985b). Georg Henrik von Wright: Of Human Freedom. En McMurrin (1985).

Von Wright (1986). Georg Henrik von Wright: Rationality: Means and End. Epistemologia 9 (1986).

Von Wright (1987). Georg Henrik von Wright: Truth-Logics. Logique et Analyse 30 (1987).

Von Wright (1988). Georg Henrik von Wright: Action Logic as a Basis of Deontic Logic. In Bernardo (1988).

Von Wright (1991). Georg Henrik von Wright: Is there a Logic of Norms? Ratio Juris 4 (1991).

Von Wright (1994). Georg Henrik von Wright: Normen, Werte und Handlungen. Frankfurt 1994.

Von Wright (1997). Georg Henrik von Wright: Ought to Be - Ought to Do. Garzon Valdés et al. (1997). 
Von Wright (1998a). Georg Henrik von Wright: Ought to Be - Ought to Do

Von Wright (1998b). Georg Henrik von Wright: In the Shadow of Descartes. Dordrecht 1998.

Von Wright/Meggle (1989). Georg Henrik von Wright und Georg Meggle: Das Verstehen von Handlungen (Münsteraner Disputation). Rechtstheorie 20, 1989; reprinted in von Wright (1994). 